\title{
Research on Peak-detection Algorithm for High-precision Demodulation System of Fiber Bragg Grating
}

\author{
Peng Wang ${ }^{1,2 *}, \mathrm{Xu} \mathrm{Han}^{1}$, Simin Guan ${ }^{1}$, Hong Zhao ${ }^{2}$ and Minglei Shao ${ }^{1}$ \\ ${ }^{1}$ College of Electrical and Electronic Engineering, Harbin University of Science and \\ Technology, Harbin, 150080, China \\ ${ }^{2}$ Key Laboratory of Engineering Dielectrics and Its Application, Ministry of \\ Education of China, Harbin, 150080, China \\ wpkunpeng@163.com
}

\begin{abstract}
In order to improve the detection accuracy of wavelength, the filtering and curve fitting technologies were applied in the FBG wavelength demodulation system based on tunable F-P filter. These methods could realize the accurate peak-location of output signals of the photo detector. According to the characteristics of noise, the FIR low-pass filter was designed to filter the obtained light power signals so as to provide the input signals with high SNR for the peak-detection algorithms. By analyzing and comparing several typical peak-searching algorithms, the algorithm of Gauss formula nonlinear curve fitting (L-M) was chosen to fit the digitized light power signals. The experimental results show that L-M fitting algorithm reduces the mean square error by $7.5 \%$ compared with the Gauss fitting algorithm. For the Gauss signal in the wavelength demodulation system designed in the paper, the L-M algorithm has lower mean square error than other peak-searching algorithms. This algorithm is suitable for FBG wavelength demodulation system based on tunable F-P filter. It can efficiently raise the accuracy of wavelength demodulation system.
\end{abstract}

Keywords: fiber Bragg grating, tunable Fabry-Perot filter, peak-detection algorithm, Levenberg-Marquardt algorithm

\section{Introduction}

Fiber Bragg grating (FBG) is a new type of optical fiber sensor [1]. It has many advantages, such as small volume, light weight, high temperature resistance, corrosion resistance, insensitivity to electromagnetic interference, easy to be embedded in intelligent structure and composed of distributed measurement network and so on [2-3]. So it is widely applied in structural health monitoring, aerospace, petrochemical and other fields [4-6]. The grating period and refractive index will alter with changes of external environment. As a result, the central wavelength of FBG will drift. By detecting the shift in the Bragg wavelength due to some physical quantities, indirect measurement of these parameters can be completed [7-8]. The tuning-wavelength method and detecting-wavelength technology are two important aspects of research on FBG. The former is sensing process of light and the latter is demodulation process of signals. The precision of FBG sensors is mainly determined by them. Among them, the detection resolution of demodulation system and peak-searching errors are important factors to determine precision [9]. In practical application, the accurate position of center wavelength can be obtained by digitalizing optical power signals and regression analysis using curve fitting techniques. These methods can effectively improve the detection accuracy [10-11]. Signal-to-noise ratio (SNR) of the input signal is the most 
important factor to affect errors of peak-searching algorithms [12]. In this paper, the wavelength demodulation system based on tunable F-P filter was introduced. Firstly, the acquired light power signals were processed by filters so as to provide input signals with high SNR for the peak-searching algorithm. After analyzing and comparing several available peak-searching algorithms, the algorithm of Gauss formula nonlinear curve fitting was chosen. It can effectively control the errors and improve the detection precision of the demodulation system.

\section{Tunable F-P Filter Demodulation System}

Demodulation methods for FBG sensing system mainly include narrow-band laser scanning method, interference method, filtering method, dispersion method, and tunable F-P filter method. In these methods, tunable F-P filter technology is more advantageous. It has advantages of high sensitivity, wide tuning range, and suitable for multi-point measurement. The wavelength demodulation system based on tunable F-P filter is designed as shown in Figure 1.

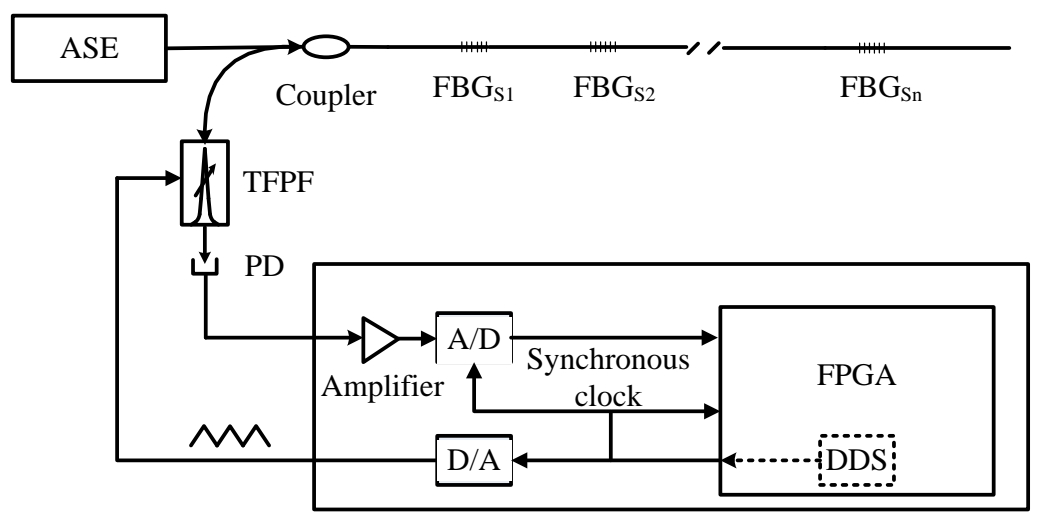

Figure 1. Wavelength Demodulation System based on Tunable F-P Filter

Broadband light source is connected with the sensing grating array by coupler. Each FBG sensor is in series with other FBG sensors on a single fiber. They have different center wavelengths and different dynamic ranges. The light emitted by broadband optical source passes coupler, then incidences FBG sensors. The light wave, which meets the Bragg equation of some FBG sensor, is reflected back into the tunable F-P filter via the coupler. The tunable F-P filter is scanning periodically under driving by the triangular wave. Its scanning range covers the center wavelengths and maximum dynamic ranges of all FBG sensors. The optical wave meeting peak transmission conditions will be transmitted out at different time. Then it is converted into voltage signal by photoelectric detector. Subsequently, the signal is transformed into digital signal by A/D conversion circuit after amplification. The acquisition circuits of PD output, triangular wave signal generator and control units are controlled by the signal processing unit. The driving voltage of PZT and acquisition circuits of PD output can be synchronized using the synchronous clock. Then the wavelength information figured up in signal processing unit is uploaded to computer and done further processing. 


\section{Design of Filter Algorithm}

In order to improve the locating accuracy of peak value, noise must be removed from useful signals. Since the noise in the FBG demodulation system was mainly Gauss white noise, the FIR low-pass filter was designed to process collected signals. The sampling frequency was set to $4 \mathrm{MHz}$. The cut-off frequency of the ideal low-pass filter was $5 \mathrm{KHz}$ and the order is 32 . According to performance of the filter, the function simulation was done in MTALAB. The center wavelength of the FBG is $1563.807 \mathrm{~nm}$. The result is demonstrated in Figure 2. It can clearly be sawn that the signals become smoother due to filtering most of noise.
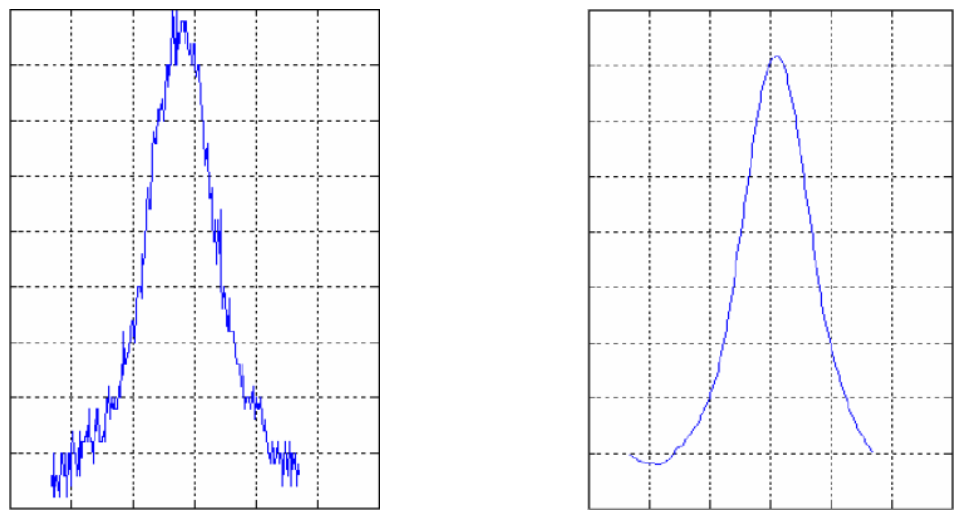

Figure 2. Primitive Signal and Filtered Signal

\section{Peak-searching Algorithm}

\subsection{Direct Peak-detection Algorithm}

The algorithm of direct peak-detection is relatively simple. It means to directly calculate the maximum peak position of the collected data. In the case of low noise, it can directly obtained wavelength value corresponding to maximum peak position. This wavelength value is the center wavelength of the FBG. But it will lead to large error that the noise is high, especially when the power around the peak point strongly fluctuates in reflection spectral.

\subsection{Gauss Curve Fitting Algorithm}

The algorithm of Gauss curve fitting is to process collected data by Gauss polynomial transform. After being fitting according to general polynomial, the peak position can be determined. The reflection spectrum of FBG may be described by Gauss function as formula (1).

$$
I(\lambda)=I_{0} \exp \left[-4 \ln 2\left(\frac{\lambda-\lambda_{B}}{\Delta \lambda_{B}}\right)^{2}\right]
$$

Where $I_{0}$ is the amplitude of the refection spectrum intensity $I, \lambda_{B}$ is Bragg wavelength of the FBG, $\Delta \lambda_{B}$ is $3 \mathrm{~dB}$ bandwidth of the refection spectrum. Take logarithm on both sides of formula (1) respectively, and the formula (2) is got.

$$
\ln I(\lambda)=-\left(4 \ln 2 \frac{\lambda_{B}^{2}}{\Delta \lambda_{B}^{2}}-\ln I_{0}\right)+8 \ln 2 \frac{\lambda_{B}^{2}}{\Delta \lambda_{B}^{2}} \lambda-4 \ln 2 \frac{1}{\Delta \lambda_{B}^{2}} \lambda^{2}
$$


Where $\quad P(\lambda)=\ln I(\lambda) \quad, \quad a_{0}=-\left(4 \ln 2 \frac{\lambda_{B}^{2}}{\Delta \lambda_{B}^{2}}-\ln I_{0}\right) \quad, \quad a_{1}=8 \ln 2 \frac{\lambda_{B}}{\Delta \lambda_{B}^{2}} \quad$, $a_{2}=-4 \ln 2 \frac{1}{\Delta \lambda_{B}^{2}}$. And the formula (1) becomes formula (3).

$$
P(\lambda)=a_{0}+a_{1} \lambda+a_{2} \lambda^{2}
$$

Formula (3) is a typical form of quadratic polynomial fitting. Assume there is n sample points $\left(\lambda_{i}, I_{i}\right),(\mathrm{i}=1,2 \ldots \mathrm{n})$. According to the least squares method, the squared deviations sum $\mathrm{S}$ of formula (3) is described as formula (4).

$$
S=\sum_{i=1}^{n}\left(I_{i}-P\left(\lambda_{i}\right)\right)^{2}
$$

When $\mathrm{S}$ is taken the minimum, the numbers of $a_{0}, a_{1}$ and $a_{2}$ are obtained. And then the wavelength of the FBG is described as formula (5).

$$
\lambda_{B}=-a_{1} / 2 a_{2}
$$

\subsection{Gauss Formula Nonlinear Curve Fitting Algorithm}

The algorithm of Gauss formula nonlinear curve fitting (Levenberg-Marquardt) is a kind of method for solving nonlinear optimization. It is a typical method for leastsquare optimal solution. Actually, this algorithm can be taken as a modified Gauss Newton method. It combines the steepest descent method (also called gradient method) with Gauss Newton method. So it contains the global features of the steepest descent method and the local characteristics of Gauss Newton method. And its convergence rate is faster than the steepest descent method. In practical application, the L-M algorithm is better able to resist influence of noise. Even if the initial values set deviates from final solutions, it can accurately get the optimal solution.

The FBG reflection spectrum is Gauss shape and the model is described as formula (6).

$$
M(\mathrm{x}, t)=a \exp \left[-\left(\frac{t-b}{c}\right)^{2}\right]
$$

Where $\mathrm{x}=[a, b, c]^{T}$. Assume there is a $x^{+}$to satisfy formula (7).

$$
\mathrm{y}_{i}=M\left(x^{+}, t_{i}\right)+\varepsilon_{i}
$$

Where $\varepsilon_{\mathrm{i}}$ is the measurement error. The Error of $\mathrm{f}_{i}(x)$ and $F(x)$ ( the quadratic sum of error) are respectively described as formula (8) and formula (9).

$$
\begin{gathered}
f_{i}(\mathrm{x})=y_{i}-M\left(x, t_{i}\right) \\
F(\mathrm{x})=\frac{1}{2} \sum_{1}^{m}\left(f_{i}(x)\right)^{2}=\frac{1}{2}\|f(x)\|^{2}=\frac{1}{2} f(x)^{T} f(x)
\end{gathered}
$$


The target is to find such a group of parameters as $\mathrm{x}^{*}$, which make $F(x)$ have minimum value. It means $F\left(x^{*}\right) \leq F(x)$ and $\left\|\mathrm{x}-x^{*}\right\| \leq \delta$, where $\delta$ is a very small positive number. The initial parameters $(\mathrm{a}, \mathrm{b}, \mathrm{c})$ were respectively set to $10.1,0.04352,0.0008807$. The maximum number of iterations is 200 and the damping coefficient is 0.01 .

\section{Implementation of Filtering and Peak-searching Algorithm on FPGA}

In order to ensure the system to reliably run in real time, FPGA has been chosen as the core part of the lower machine. The whole structure of signal processing unit is shown in Figure 3.

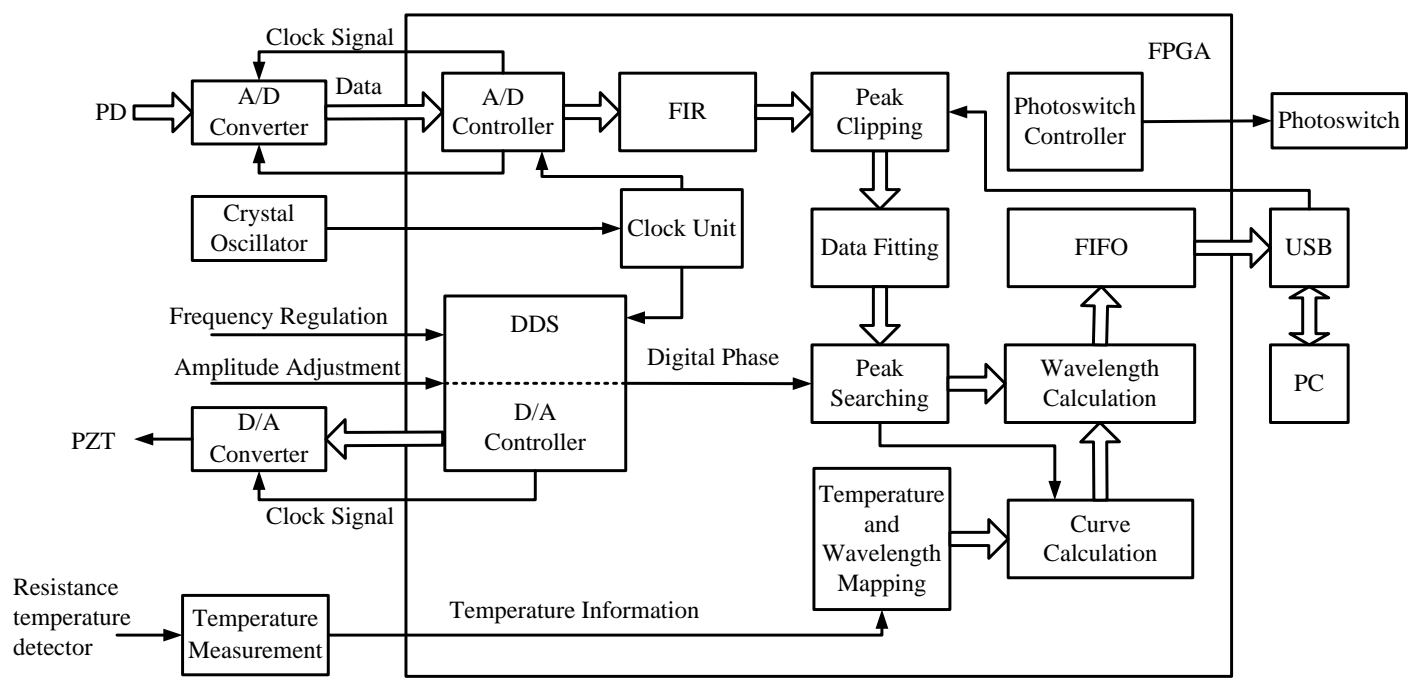

Figure 3. Structure of Signal Processing Unit

Since FPGA has good characteristics of high integration and speed, it is considered to have better real-time performance to realize filtering and peak-searching algorithm on FPGA than on PC. Firstly, the signal of the PD output was changed to digital signals by AD. And then the FIR low-pas filter was applied to filter the AD output signals so as to provide the input signals with high SNR for the peak-detection algorithms. In order to reduce useless data and running time, peaks-separating and amplitude-cutting-off was carried out to process data according to set threshold. The data above the threshold was processed by selected curve fitting algorithm. In this way, it is more accurate to find the peak moment.

In addition, FPGA can divide the frequency of external crystal oscillator using inner PLL to provide clocks for inner circuit units. It can control AD to convert the output signals of PD into digital signals. It can generate the triangular wave driving signal of PZT and control signal output of DA.

After being processed by FPGA, the data are uploaded to upper computer by USB to do further analysis, store and display.

\section{Experimental Result and Analysis}

ASE broadband light source was used in experiments. The scanning frequency of F-P cavity is $100 \mathrm{~Hz}$. The temperature around the grating array kept constant. The captured signals were filtered and fitted in order. For ten groups of experimental data, the 
locations of peak voltage were detected respectively by the direct peak-detection algorithm, the Gauss curve fitting algorithm and L-M fitting algorithm. The distribution of absolute errors is shown in Figure 4. The mean square errors of three algorithms are listed in Table 1. The experimental results demonstrate that the curve fitted by L-M algorithm is closer to fact among three algorithms, and the L-M algorithm has the lowest mean square error for locating peak value. It can raise the precision of the demodulation system, and is a practical method.

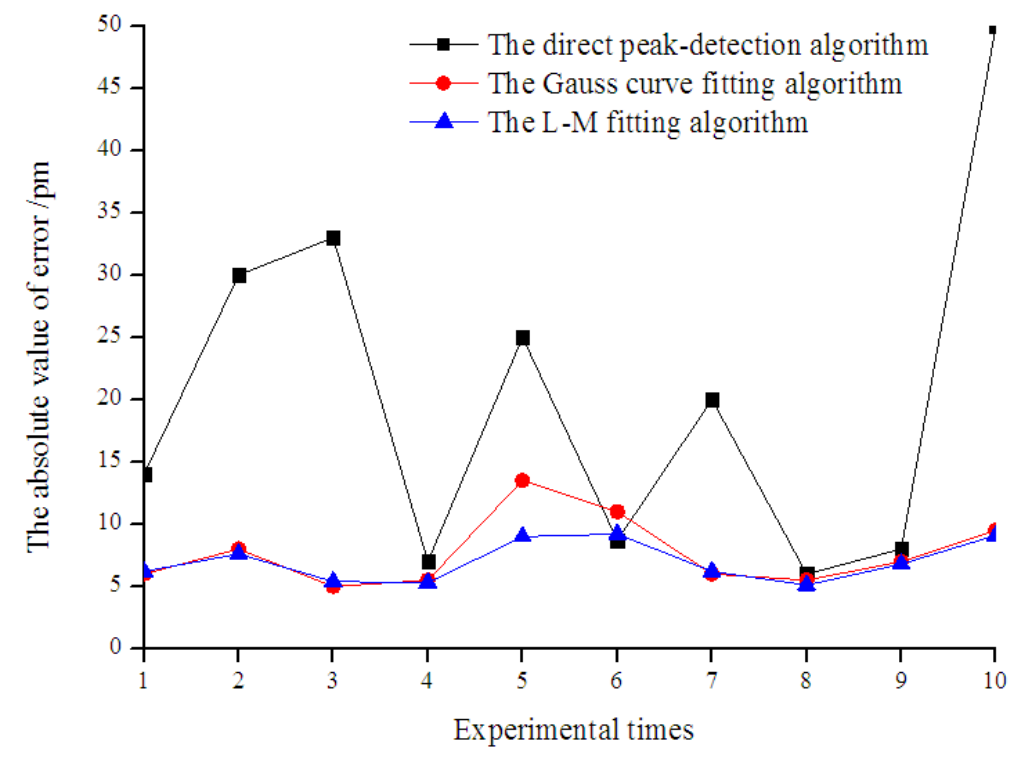

Figure 4. Experimental Result

Table 1. Mean Square Errors of Three Curve Fitting Algorithms

\begin{tabular}{c|c|c}
\hline $\begin{array}{c}\text { Direct peak-detection } \\
\text { algorithm }\end{array}$ & $\begin{array}{c}\text { Gauss curve fitting } \\
\text { algorithm }\end{array}$ & $\begin{array}{c}\text { L-M fitting } \\
\text { algorithm }\end{array}$ \\
\hline $19.69 \mathrm{pm}$ & $7.18 \mathrm{pm}$ & $6.64 \mathrm{pm}$ \\
\hline
\end{tabular}

\section{Conclusion}

According to the features of noise in FBG wavelength demodulation system, the low pass filter and curve fitting technique were applied to process signals. Firstly, the signals were processed by filters. Then curve fitting was carried out. This method can be more accurate on location of the peak. The experimental results show that L-M algorithm is more effective than the algorithm of gauss fitting in solving the least squares problem. For the design of wavelength demodulation system designed in this paper, the L-M algorithm has minimal mean square error among several available peaksearching algorithms used to fit the signals, whose shapes are similar to the Gauss signal. Being compared with Gauss fitting algorithm, it reduces the mean square error by $7.5 \%$. Theoretical simulation and experimental results demonstrate that this algorithm is applicable to FBG wavelength demodulation system based on tunable F-P filter. It can effectively improve the demodulation precision. 


\section{Acknowledgements}

This work was supported by Educational Commission of Heilongjiang (12521106).

\section{References}

[1] X.-C. Ma and Z.-A. Zhou, J. Journal of Optoelectronics, Laser, vol. 7, no. 24, (2003).

[2] G.-W. Li, H.-F. Pei and J.-H. Yin, "J. Measurement Journal of the International Measurement Confederation”, vol. 1, no. 49, (2014).

[3] J.-Z. Li, B.-C. Sun and Y.-L. Du, "J. Optoelectronics Letters", vol. 1, no. 10, (2014).

[4] H.-P. Wang, Y.-Q. Tan, Z.-J. Dong, "J. Journal of Optoelectronic", Laser, vol. 11, no. 24, (2013).

[5] J. Jin, S. Lin and X.-Y. Ye, ”J. Optik”, vol. 1, no. 125, (2014).

[6] J.-W. Fu, L.-Z. Xiao and Y.-Z. Zhang, "J. Optical Technique", vol. 6, no. 32, (2006).

[7] Q. Lu, L.-J. Cai and Z.-Y. Li, "J. Journal of Optoelectronics”, Laser, vol. 7, no. 23, (2012).

[8] Y.-L. Yu, X.-W. Wang and H. Wang, "J. Acta Photonica Sinca", vol. 11, no. 41, (2012).

[9] J.-F. Jiang, T.-G. Liu and K. Liu, "J. Optical Engineering”, vol. 3, no. 51, (2012).

[10] H.-H. Zhu, H.-K. Qin and M. Zhang, "J. Chinese Journal of Lasers", vol. 6, no. 35, (2008).

[11] F.-G. Wu, Q.-S. Zhang and D.-S. Jiang, "J. Jounal of Wuhan University of Technology", vol. 12, no. 29, (2007).

[12] F. Grzegorz and N. Pawel, R. J. McDonald, "J. Optical Engineering”, vol. 3, no. 46, (2007). 
International Journal of Hybrid Information Technology Vol. 7, No. 6 (2014) 